\title{
Relay Selection for MIMO Two-Way Relay Networks with Spatial Multiplexing
}

\author{
Shashindra Silva*, Gayan Amarasuriya ${ }^{\dagger}$, Chintha Tellambura* and Masoud Ardakani* \\ *Department of Electrical and Computer Engineering, University of Alberta, Edmonton, AB, Canada T6G 2V4 \\ Email: \{jayamuni,chintha, ardakani\}@ece.ualberta.ca \\ ${ }^{\dagger}$ Department of Electrical Engineering, Princeton University, Princeton, NJ, USA 08544 \\ Email:gbaduge@princeton.edu
}

\begin{abstract}
Relay selection strategies help to improve spectral and energy efficiencies, to enhance transmission robustness, or to reduce latency in cooperative networks. A novel relay selection strategy, which maximizes the overall achievable sum rate, is proposed and analyzed for multiple-input multiple-output (MIMO) amplifyand-forward two-way relay networks with spatial multiplexing. Interestingly, this strategy amounts to maximizing the minimum of the determinant of the Wishart matrices from the relays to the two user nodes. The performance of this strategy is investigated by deriving the average sum rate approximations in closed-form. Our analysis and numerical results reveal that the proposed relay selection scheme provides substantial sum rate improvements.
\end{abstract}

\section{INTRODUCTION}

In two-way relay networks (TWRNs), user nodes with bidirectional data-flows mutually exchange their data signals via relay nodes [1], [2]. This requires just two channel-uses for the twoway mutual data exchange unlike the four channel-uses required by the one-way relay networks (OWRNs) with half-duplex nodes. Consequently, the spectral efficiency of TWRNs are twice that of OWRNs, and thus, they are currently being examined for next generation wireless communication standards, including Longterm evolution-advanced (LTE-A) [3]. One of the key technology enabling the reliability and data rate goals of these standards is the multiple-input multiple-output (MIMO) technology.

MIMO wireless technology can provide significant performance improvements in terms of data rates and link-reliability due to spatial multiplexing gains and diversity gains [4]. Both spatial multiplexing and diversity benefits can indeed be achieved subjected to a fundamental trade-off whenever efficient spacetime code designs are employed [5]. In particular, MIMO relays can improve the performance of TWRNs [2], [6], [7].

Relay selection is an important concept in cooperative relay networks, in which, out of all potential relays, only a subset of one or more are selected to relay the signals between a transmitter (Tx) node and a receiver $(\mathrm{Rx})$ node [8]. Relay selection criteria can be designed to improve spectral and energy efficiencies, to enhance transmission robustness, or to reduce latency in multirelay cooperative networks [9].

Thus, MIMO relay selection can be used to improve performance of TWRNs [2], [6]. Thus, a novel relay selection strategy for MIMO amplify-and-forward (AF) TWRNs with zero-forcing (ZF) transmissions for maximizing the achievable sum rate is developed here.

Previous research on relay selection for MIMO TWRNs: Joint relay and antenna selection strategies over Rayleigh fading are investigated in [2]. In [2], transmission impairments including the feedback-delay effect and spatially correlated fading on the performance of antenna selection are studied, and the amount of performance degradation is quantified. A joint beamforming and relay selection strategy to maximize the end-to-end signalto-noise ratio (SNR) is developed in [7]. In particular, in [7], the overall outage probability is derived in closed-form, and thereby, quantifying the achievable diversity order. However, it must be stressed that the relay selection strategies of [2] and [7] have been developed for a single end-to-end spatial data-stream. Thus, relay selection for MIMO TWRNs with multiple end-to-end spatial data-streams (i.e., with spatial multiplexing) has not yet been investigated.

Previous research on relay selection for SISO TWRNs: For the sake of completeness, several important prior related studies on relay selection for single-antenna TWRNs are next summarized. In [10], single and multiple relay selection schemes are developed and analysed for AF TWRNs. In [11], an optimal relay selection scheme is developed with full-duplex nodes based on maximizing the effective signal-to-interference-plus-noise ratio (SINR). Moreover, in [12], [13], the relay selection schemes are studied for single-antenna TWRNs with physical-layer network coding. References [14]-[16] develop relay selection strategies for decode-and-forward (DF) TWRNs.

Motivation and our contribution: Thus, in the extensive wireless relay literature, we find that several MIMO TW relay selection strategies for single end-to-end spatial data streams have been treated in [2] and [7] only. However, these strategies are more suited for practical scenarios where the MIMO wireless channels are ill-conditioned (due to lack of rich scattering) or if the transmission reliability via the diversity gains is traded off for the data rates via spatial multiplexing gains. In this context, design and analysis of relay selection strategies for MIMO TWRNs with spatial multiplexing (i.e., with more than one end-to-end spatial data-streams) is not available. This observation thus motivates our work, which fills the aforementioned gap by proposing and analyzing a novel relay selection strategy for MIMO TWRNs with spatial multiplexing.

Our proposed strategy is developed based on maximizing the achievable sum rate. Thus, it selects the relay which maximizes the minimum of the determinants of the Wishart matrices of the selected relay to the two user nodes. The exact derivation of the ergodic sum rate appears mathematically intractable due to statistical dependence of data substream SNRs belonging to a 
given user node. We thus derive the closed-form average sum rate approximations. Rigorous simulation results are presented to investigate/compare the performance of the proposed selection strategy and to verify our analysis.

Notation: $\mathbf{Z}^{H}, \mathbf{Z}^{T}$, and $[\mathbf{Z}]_{k, k}$ denote the Hermitian-transpose, transpose, and the $k$ th diagonal element of the matrix, $\mathbf{Z}$, respectively. $E_{1}(z)$ is the exponential integral function for the positive values of the real part of $\mathrm{z}$ [17, Eqn. (8.211)], and $\Gamma(a, z)$ is the upper incomplete Gamma function [17, Eqn. (8.350.2)].

\section{SyStem, CHANNEL, AND SIGNAL MODEL}

This section presents the system, channel, and signal model of the MIMO ZF TWRN. Further, the exact end-to-end SNRs at the user nodes are derived, and thereby the upper bound of the SNRs are obtained as well.

\section{A. System and channel model}

The system model consists of two user nodes $\left(U_{1}\right.$ and $\left.U_{2}\right)$ and $L$ relay nodes $\left(R_{l}\right.$ for $\left.l \in\{1, \cdots, L\}\right)$. User node $U_{i}$ is equipped with $N_{i}$ antennas for $i \in\{1,2\}$, and the $l$ th relay node has $N_{R_{l}}$ antennas. All nodes are assumed half-duplex terminals, and all channel amplitudes are assumed independently distributed, frequency flat-Rayleigh fading. Thus, the channel matrix from $U_{i}$ to $R_{l}$ can be defined as $\mathbf{F}_{i, l}=\tilde{\mathbf{F}}_{i, l} \mathbf{D}_{i, l}^{1 / 2}$, where $\tilde{\mathbf{F}}_{i, l} \sim \mathcal{C} \mathcal{N}_{N_{R_{l}} \times N_{i}}\left(\mathbf{0}_{N_{R_{l}} \times N_{i}}, \mathbf{I}_{N_{R_{l}}} \otimes \mathbf{I}_{N_{i}}\right)$ captures the fast fading and $\mathbf{D}_{i, l}=\eta_{i, l} \mathbf{I}_{N_{R_{l}}}$ accounts for the pathloss. The channel coefficients are assumed to be fixed during two consecutive timeslots, and hence, the channel matrix from $R_{l}$ to $U_{i}$ can be written as $\mathbf{F}_{l, i}=\mathbf{F}_{i, l}^{T}$ by using the reciprocity property of wireless channels. The additive noise at all the receivers is modelled as complex zero mean additive white Gaussian (AWGN) noise. The direct channel between $U_{1}$ and $U_{2}$ is assumed to be unavailable due to large pathloss and heavy shadowing effects [1], [6].

\section{B. Signal model}

During two time-slots, $U_{1}$ and $U_{2}$ exchange their signal vectors $\left(\mathbf{x}_{1}\right.$ and $\mathbf{x}_{2}$ ) by selecting one of the available $L$ relays. Here, the selected relay is denoted as $R_{l}$ for the sake of the exposition. In the first time-slot, $U_{1}$ and $U_{2}$ transmit $\mathbf{x}_{1}$ and $\mathbf{x}_{2}$, respectively, towards $R_{l}$ by employing transmit-ZF precoding over the multiple access channel ${ }^{1}$. The received signal at $R_{l}$ can then be written as

$$
\mathbf{y}_{R_{l}}=g_{1, l} \mathbf{F}_{1, l} \mathbf{W}_{T_{1, l}} \mathbf{x}_{1}+g_{2, l} \mathbf{F}_{2, l} \mathbf{W}_{T_{2, l}} \mathbf{x}_{2}+\mathbf{n}_{R_{l}},
$$

where the $N_{R_{l}} \times 1$ signal vector $\mathbf{x}_{i}$ satisfies $\mathcal{E}\left[\mathbf{x}_{i} \mathbf{x}_{i}^{H}\right]=\mathbf{I}_{N_{R_{l}}}$ for $i \in\{1,2\}$ and $l \in\{1, \cdots, L\}$. Thus, the $N_{i} \times 1$ precodedtransmit signal vector at $U_{i}$ is given by $\mathbf{W}_{T_{i, l}} \mathbf{x}_{i}$. In (1), $g_{i, l}$ is the power normalizing factor at $U_{i}$ and is designed to constrain its long-term transmit power as follows [6]:

$g_{i, l}=\sqrt{\mathcal{P}_{i} / \operatorname{Tr}\left(\mathcal{E}\left[\mathbf{W}_{T_{i, l}} \mathbf{W}_{T_{i, l}}^{H}\right]\right)}=\sqrt{\mathcal{P}_{i} \eta_{i, l}\left(N_{i}-N_{R_{l}}\right) / N_{R_{l}}},(2)$

\footnotetext{
${ }^{1}$ In order to use transmit-ZF at $U_{1}$ and $U_{2}$, the constraint $\min \left(N_{1}, N_{2}\right) \geq$ $\max _{l \in\{1, \cdots, L\}} N_{R_{l}}$ needs to be satisfied.
}

where $\mathcal{P}_{i}$ is the transmit power at $U_{i}{ }^{2}$. Further, in (1), $\mathbf{n}_{R_{l}}$ is the $N_{R_{l}} \times 1$ zero mean AWGN vector at $R_{l}$ satisfying $\mathcal{E}\left(\mathbf{n}_{R_{l}} \mathbf{n}_{R_{l}}^{H}\right)=$ $\mathbf{I}_{N_{R_{l}}} \sigma_{R_{l}}^{2}$, and $\mathbf{W}_{T_{i, l}}$ is the transmit-ZF precoding matrix at $U_{i}$ given by [18]

$$
\mathbf{W}_{T_{i, l}}=\mathbf{F}_{i, l}^{H}\left(\mathbf{F}_{i, l} \mathbf{F}_{i, l}^{H}\right)^{-1} .
$$

In the second time-slot, $R_{l}$ amplifies $\mathbf{y}_{R_{l}}$ and broadcasts this amplified-signal towards both user nodes. Each user node then receives its signal vector by using the corresponding receive-ZF detector as follows:

$$
\mathbf{y}_{U_{i, l}}=\mathbf{W}_{R_{i, l}}\left(G_{l} \mathbf{F}_{l, i} \mathbf{y}_{R_{l}}+\mathbf{n}_{i}\right),
$$

where $G_{l}$ is the amplification factor at $G_{l}$ and can be defined as

$$
G_{l}=\sqrt{\mathcal{P}_{R_{l}} /\left(g_{1, l}^{2}+g_{2, l}^{2}+\sigma_{R_{l}}^{2}\right)},
$$

where $g_{l, i}$ is defined in (2), $\sigma_{R_{l}}^{2}$ is the variance of the additive noise at $R_{l}$, and $\mathcal{P}_{R_{l}}$ is the transmit power at $R_{l}$. Moreover, in (4), $\mathbf{F}_{l, i}=\mathbf{F}_{i, l}^{T}$, and $\mathbf{n}_{i}$ is the $N_{i} \times 1$ zero mean AWGN at $U_{i}$ satisfying $\mathcal{E}\left(\mathbf{n}_{i} \mathbf{n}_{i}^{H}\right)=\mathbf{I}_{N_{i}} \sigma_{i}^{2}$. Besides, $\mathbf{W}_{R_{i, l}}$ is the receive-ZF matrix at $U_{i}$ and can be written as [18]

$$
\mathbf{W}_{R_{i, l}}=\left(\mathbf{F}_{l, i}^{H} \mathbf{F}_{l, i}\right)^{-1} \mathbf{F}_{l, i}^{H} .
$$

\section{Exact end-to-end SNR}

In this subsection, the exact end-to-end SNR of the $k$ th data substream for $k \in\left\{1, \cdots, N_{R_{l}}\right\}$ is derived by using the signalling model presented in Section II-B. To this end, by substituting (1), (3), and (6) into (4), the received signal vector at $U_{i}$ can be written in an alternative form as follows:

$$
\mathbf{y}_{U_{i, l}}=G_{l}\left(g_{i, l} \mathbf{x}_{i}+g_{i^{\prime}, l} \mathbf{x}_{i^{\prime}}+\mathbf{n}_{R_{l}}\right)+\tilde{\mathbf{n}}_{i},
$$

where $\left\{i, i^{\prime}\right\} \in\{\{1,2\},\{2,1\}\}$. Further, $\tilde{\mathbf{n}}_{i}$ is the filtered, colored noise and is given by $\tilde{\mathbf{n}}_{i}=\mathbf{W}_{R_{i, l}} \mathbf{n}_{i}$. Next, by using selfinterference cancellation to (7), the signal vector of $U_{i^{\prime}}$ received at $U_{i}$ can be extracted as follows:

$$
\tilde{\mathbf{y}}_{U_{i, l}}=G_{l}\left(g_{i^{\prime}, l} \mathbf{x}_{i^{\prime}}+\mathbf{n}_{R_{l}}\right)+\tilde{\mathbf{n}}_{i}
$$

By using (8), the post-processing end-to-end SNR of the $k$ th data substream at $U_{i}$ can be derived as

$$
\gamma_{U_{i, l}^{(k)}}^{(k)}=\frac{G_{l}^{2} g_{i^{\prime}, l}^{2}}{G_{l}^{2} \sigma_{R_{l}}^{2}+\frac{\sigma_{i}^{2}}{\eta_{i, l}}\left[\left(\tilde{\mathbf{F}}_{l, i}^{H} \tilde{\mathbf{F}}_{l, i}\right)^{-1}\right]_{k, k}},
$$

where $\left\{i, i^{\prime}\right\} \in\{\{1,2\},\{2,1\}\}, k \in\left\{1, \cdots, N_{R_{l}}\right\}$, and $l \in$ $\{1, \cdots, L\}$. By substituting $G_{l}$ (5) and $g_{i^{\prime}, l}$ (2) into (9), the end-to-end SNR in (9) can be written in a more insightful form as shown in (10) on the top of the next page. Here in (10), $\bar{\gamma}_{i, l} \triangleq \mathcal{P}_{i} \eta_{i, l} / \sigma_{R_{l}}^{2}, \bar{\gamma}_{l, i} \triangleq \mathcal{P}_{R_{l}} \eta_{i, l} / \sigma_{i}^{2}, i \in\{1,2\}, i^{\prime} \in\{1,2\}$, $l \in\{1, \cdots, L\}$ and $i \neq i^{\prime}$.

Remark II.1: It is worth noting that $\gamma_{U_{1, l}^{(k)}}$ and $\gamma_{U_{2, l}^{(k)}}$ for $k \in$ $\left\{1, \cdots, N_{R_{l}}\right\}$ of (10) are statistically independent for a given

\footnotetext{
${ }^{2}$ To obtain non-zero $g_{i, l}$, the constraint $N_{i} \neq N_{R_{l}}$ needs to be satisfied. Thus, in order to employ transmit-ZF at $U_{1}$ and $U_{2}$, and the long-term transmit power constraint at $R_{l}$, the constraint $\min \left(N_{1}, N_{2}\right)>\max _{l \in\{1, \cdots, L\}} N_{R_{l}}$ should be satisfied.
} 


$$
\gamma_{U_{i, l}^{(k)}}=\frac{\left(N_{i^{\prime}}-N_{R_{l}}\right) \bar{\gamma}_{i^{\prime}, l} \bar{\gamma}_{l, i}}{N_{R_{l}} \bar{\gamma}_{l, i}+\left(\left(N_{i^{\prime}}-N_{R_{l}}\right) \bar{\gamma}_{i^{\prime}, l}+\left(N_{i}-N_{R_{l}}\right) \bar{\gamma}_{i, l}+N_{R_{l}}\right)\left[\left(\tilde{\mathbf{F}}_{l, i}^{H} \tilde{\mathbf{F}}_{l, i}\right)^{-1}\right]_{k, k}}
$$

$k$ and $l$. However, post-processing SNRs of multiple substreams belonging to a given user node are correlated, i.e., $\gamma_{U_{i, l}^{(k)}}$ and $\gamma_{U_{i, 1}^{\left(k^{\prime}\right)}}$ are correlated for a given $i$ and $l$. Due to this correlation effect, derivation of the probability distributions of the SNR of the smallest data substream appears mathematically intractable, and hence, a SNR upper bound is derived in the next subsection.

\section{Upper bound on the SNR of the smallest data substream and its probability distribution}

In this subsection, an upper bound on the end-to-end SNR of the smallest data substream is derived. In particular, this SNR upper bound is used in deriving the sum-rate in closed-form in the sequel. The maximum diagonal element of $\left(\mathbf{F}_{l, i}^{H} \mathbf{F}_{l, i}\right)^{-1}$ can be lower bounded by any of its diagonal elements [6]. Thus, the SNR of the smallest substream of $U_{i}$ for $i \in\{1,2\}$ can be upper bounded as

$$
\gamma_{U_{i, l}^{\min }}=\min _{k \in\left\{1 \cdots N_{R_{l}}\right\}^{(i, l}} \gamma_{U^{(k)}} \leq \gamma_{U_{i, l}^{\min }}^{\mathrm{ub}}=\frac{\alpha_{i, l}}{\beta_{i, l}+\zeta_{i, l}\left[\left(\tilde{\mathbf{F}}_{l, i}^{H} \tilde{\mathbf{F}}_{l, i}\right)^{-1}\right]_{k, k}},
$$

where $\alpha_{i, l}=\left(N_{i^{\prime}}-N_{R_{l}}\right) \bar{\gamma}_{i^{\prime}, l} \bar{\gamma}_{l, i}, \beta_{i, l}=N_{R_{l}} \bar{\gamma}_{l, i}$, and $\zeta_{i, l}=$ $\left(N_{i^{\prime}}-N_{R_{l}}\right) \bar{\gamma}_{i^{\prime}, l}+\left(N_{i}-N_{R_{l}}\right) \bar{\gamma}_{i, l}+N_{R_{l}}$ for $i \in\{1,2\}, i^{\prime} \in\{1,2\}$, $i \neq i^{\prime}$, and $l \in\{1, \cdots, L\}$. It is worth noting that $\gamma_{U_{1, l}^{\min }}^{\mathrm{ub}}$ and are statistically independent for any $l$.

By using the distribution of the $k$ th diagonal element of the inverse Wishart matrix [19], the CDF of $\gamma_{U_{i, l}^{\min }}^{\mathrm{ub}}$ can be written as

$$
F_{\gamma_{U_{i, l}^{\min }}^{\mathrm{ub}}}(x)= \begin{cases}1-\frac{\Gamma\left(N_{i}-N_{R_{l}}+1, \frac{\zeta_{i, l} x}{\alpha_{i, l}-\beta_{i, l} x}\right)}{\Gamma\left(N_{i}-N_{R_{l}}+1\right)}, & 0<x<\frac{\alpha_{i, l}}{\beta_{i, l}} \\ 1, & x \geq \frac{\alpha_{i, l}}{\beta_{i, l}}\end{cases}
$$

It is worth noting that the SNR upper bound in (11) converges to the exact SNR when $N_{R_{l}}=1$ for $l \in\{1, \cdots, L\}$, and the tightness of this bound significantly improves as $N_{R_{l}}$ approaches unity.

\section{Problem Formulation}

This subsection formulates the proposed relay selection strategy. In MIMO TWRNs with symmetric data traffic, each user node needs to transmit its data substreams with a common rate such that these data substreams can be decoded correctly by the intended receivers, and hence, the corresponding sum rate, when the $l$ th relay is selected, can be defined as follows:

$$
\mathcal{R}_{l}=2 \min \left(\mathcal{R}_{U_{1, l}}, \mathcal{R}_{U_{2, l}}\right),
$$

where $\mathcal{R}_{U_{i, l}}$ is the sum of data substreams rates at $U_{i}$ for $i \in$ $\{1,2\}$, and can be written as

$$
\mathcal{R}_{U_{i, l}}=\frac{1}{2} \sum_{k=1}^{N_{R_{l}}} \log \left(1+\gamma_{U_{i, l}^{(k)}}\right) .
$$

It is worth noting that the factor of two appears in (13) is due to the presence of two user nodes in the TWRN. Further, the pre-log factor of one-half in (14) is due to the two time-slots used for multiple-access and broadcast phases. By first substituting (14) into (13), and then by performing several manipulations, the sum rate can be written in an alternative form as follows:

$$
\mathcal{R}_{l}=\log \left(\min \left(\prod_{k=1}^{N_{R_{l}}}\left(1+\gamma_{U_{1, l}^{(k)}}\right), \prod_{k=1}^{N_{R_{l}}}\left(1+\gamma_{U_{2, l}^{(k)}}\right)\right)\right) .
$$

By using (15), the best relay selection based on maximizing the sum rate can then be formulated as

$$
L^{*}=\underset{l \in\{1, \cdots, L\}}{\operatorname{argmax}}\left[\mathcal{R}_{l}\right]=\underset{l \in\{1, \cdots, L\}}{\operatorname{argmax}}\left[\min \left(\prod_{k=1}^{N_{R_{l}}} \gamma_{U_{1, l}^{(k)}}, \prod_{k=1}^{N_{R_{l}}} \gamma_{U_{2, l}^{(k)}}\right)\right],
$$

where $L^{*}$ is the index of the selected best relay. If $\mathcal{P}_{R_{1}}=\mathcal{P}_{R}$, $\sigma_{R_{1}}^{2}=\sigma_{R}^{2}$, and $\eta_{1, l}=\eta_{2, l}$ for $l \in\{1, \cdots, L\}$ and number of antennas are equal for each relay, then the relay selection strategy given in (16) can further be simplified by using (11) as

$$
L^{*}=\underset{l \in\{1, \cdots, L\}}{\operatorname{argmax}}\left[\min \left(\prod_{k=1}^{N_{R_{l}}}\left(\left[\mathbf{V}_{l, 1}^{-1}\right]_{k, k}\right)^{-1}, \prod_{k=1}^{N_{R_{l}}}\left(\left[\mathbf{V}_{l, 2}^{-1}\right]_{k, k}\right)^{-1}\right)\right]
$$

where $\mathbf{V}_{l, i}=\tilde{\mathbf{F}}_{l, i}^{H} \tilde{\mathbf{F}}_{l, i}$ for $i \in\{1,2\}$ and $l \in\{1, \cdots, L\}$. Then, we recall the inequality between the product of diagonal elements a positive-definite matrix and its determinant as follows [20]:

$$
\operatorname{det}(\mathbf{A}) \leq \prod_{i=1}^{M} a_{i i}
$$

where $\mathbf{A}$ is an $M \times M$ positive-definite matrix with the $i$ th diagonal element denoted as $a_{i i}$ for $i \in\{1, \cdots, M\}$.

By using the bound given by (18) in (17), the selection strategy of (16) can further be simplified as

$$
L^{*}=\underset{l \in\{1, \cdots, L\}}{\operatorname{argmax}}\left[\min \left(\operatorname{det}\left(\tilde{\mathbf{F}}_{l, 1}^{H} \tilde{\mathbf{F}}_{l, 1}\right), \operatorname{det}\left(\tilde{\mathbf{F}}_{l, 2}^{H} \tilde{\mathbf{F}}_{l, 2}\right)\right)\right] .
$$

Thus, as per (19), the best relay, which maximizes the sum rate, can be selected by maximizing the minimum of the determinants of the two Wishart matrices pertinent to the corresponding relay to its two user nodes.

\section{Performance Analysis}

In this section, tight approximations for the achievable ergodic sum rate are presented. The exact derivation of the ergodic sum rate appears mathematically intractable due to statistical dependence of data substream SNRs belonging to a given user node (see remark II.1). Thus, in this context, tight approximations of the ergodic sum rate can be derived to obtain useful insights. 


$$
\begin{aligned}
\overline{\mathcal{R}}_{L^{*}} \approx & \frac{N_{R}^{\max }}{\ln (2)} \sum_{m=0}^{N-N_{R}^{\max }} \sum_{n=0}^{N-N_{R}^{\max }} \frac{1}{2^{m+n} m ! n !}\left(\mathbb{J}\left(m+n, 2 \zeta_{L^{*}}, \alpha_{L^{*}}+\beta_{L^{*}}\right)-\mathbb{J}\left(m+n, 2 \zeta_{L^{*}}, \beta_{L^{*}}\right)\right. \\
& \left.-(m+n)\left(\mathbb{J}\left(m+n-1,2 \zeta_{L^{*}}, \alpha_{L^{*}}+\beta_{L^{*}}\right)-\mathbb{J}\left(m+n-1,2 \zeta_{L^{*}}, \beta_{L^{*}}\right)\right)\right) .
\end{aligned}
$$

$\mathbb{J}(x, y, z)=\int_{0}^{\infty} \lambda^{x} \exp (-\lambda) \ln (y+z \lambda) \mathrm{d} \lambda=\Gamma(x+1)\left(\ln (y)+\sum_{p=0}^{x} \frac{1}{\Gamma(x-p+1)}\left(\left(\frac{-y}{z}\right)^{x-p} \exp \left(\frac{y}{z}\right) E_{1}\left(\frac{y}{z}\right)+\sum_{q=1}^{x-p} \Gamma(q)\left(\frac{-y}{z}\right)^{x-p-q}\right)\right)$.

$\overline{\mathcal{R}}_{L^{*}} \approx \frac{N_{R}}{\ln (2)} \sum_{l=1}^{L} \sum_{k=1}^{2 l\left(N-N_{R}\right)}(-1)^{l}\left(\begin{array}{l}l \\ L\end{array}\right) \frac{\beta_{k, 2 l, N-N_{R}+1}}{(2 l)^{k}}((k \mathbb{J}(k-1,2 l \zeta, \alpha+\beta)+\mathbb{J}(k, 2 l \zeta, \beta)-k \mathbb{J}(k-1,2 l \zeta, \beta)-\mathbb{J}(k, 2 l \zeta, \alpha+\beta)))$.

To begin with, the sum of the rates of data substreams of $U_{i}$ (14) can be approximated by

$$
\mathcal{R}_{U_{i, l}}=\frac{1}{2} \sum_{k=1}^{N_{R_{l}}} \log \left(1+\gamma_{U_{i, l}^{(k)}}\right) \approx \frac{N_{R_{l}}}{2} \log \left(1+\gamma_{U_{i, l}^{\min }}\right)
$$

where $\gamma_{U_{i l}^{\min }}$ is defined in (11). By first substituting (20) into (13), and then by performing several mathematical formulations, an approximation for the sum rate for the best relay selection can then be written as follows:

$\mathcal{R}_{L^{*}} \approx\left(\max _{l \in\{1, \cdots, L\}}\left(N_{R_{l}}\right)\right) \log \left(1+\min \left(\gamma_{U_{1, L^{*}}^{\min }}, \gamma_{U_{2, L^{*}}^{\min }}\right)\right)$

By using (11), $\gamma_{U_{i, L^{*}}^{\min }}$ in $(21)$ can be approximated as

$$
\gamma_{U_{i, L^{*}}^{\min }} \approx \frac{\alpha_{i, L^{*}}}{\beta_{i, L^{*}}+\zeta_{i, L^{*}}\left[\left(\tilde{\mathbf{F}}_{L^{*}, i}^{H} \tilde{\mathbf{F}}_{L^{*}, i}\right)^{-1}\right]_{k, k}}
$$

where $k \in\left\{1, \cdots, N_{R}^{\max }\right\}$. Further, $\alpha_{i, L^{*}}, \beta_{i, L^{*}}$, and $\zeta_{i, L^{*}}$ are defined as follows:

$$
\begin{aligned}
& \alpha_{i, L^{*}}=\left(N_{i^{\prime}}-N_{R}^{\max }\right) \bar{\gamma}_{i^{\prime}, L^{*}} \bar{\gamma}_{L^{*}, i}, \quad \beta_{i, L^{*}}=N_{R}^{\max } \bar{\gamma}_{L^{*}, i}, \\
& \zeta_{i, L^{*}}=\left(N_{i^{\prime}}-N_{R}^{\max }\right) \bar{\gamma}_{i^{\prime}, L^{*}}+\left(N_{i}-N_{R}^{\max }\right) \bar{\gamma}_{i, L^{*}}+N_{R}^{\max } .
\end{aligned}
$$

Here, in (22b) and (22c), $N_{R}^{\max }=\max _{l \in\{1, \cdots, L\}} N_{R_{l}}, i \in\{1,2\}$, $i^{\prime} \in\{1,2\}$, and $i \neq i^{\prime}$.

The average sum rate approximation can next be derived by taking the expectation of $\mathcal{R}_{L^{*}}$ in (21) over the corresponding equivalent SNR, $\gamma_{\text {eq }}=\min \left(\gamma_{U_{1, L^{*}}^{\min }}, \gamma_{U_{2, L^{*}}^{\min }}\right)$, as in (23). (see Appendix $\mathrm{A}$ for the proof). Here in (23), the function $\mathbb{J}(x, y, z)$ is given by (24).

Remark IV.2: The close-form approximation of the ergodic sum rate presented in (23) is valid for different number of relay antennas $\left(N_{R_{l}}\right.$ for $\left.l \in\{1, \cdots, L\}\right)$. Nevertheless, our numerical results reveal that this approximation slightly weakens when all available relays are equipped with the same number of antennas $\left(N_{R_{l}}=N_{R}\right.$ for $\left.i \in\{1, \cdots, L\}\right)$. Thus, for this case, an tight ergodic sum rate approximation can next be derived explicitly as shown in (25). (see Appendix B for the proof). Here, in (25), $\alpha=$ $\alpha_{i, l}, \zeta=\zeta_{i, l}$, and $\beta=\beta_{i, l}$ for all $i \in\{1,2\}$ and $l \in\{1, \cdots, L\}$.

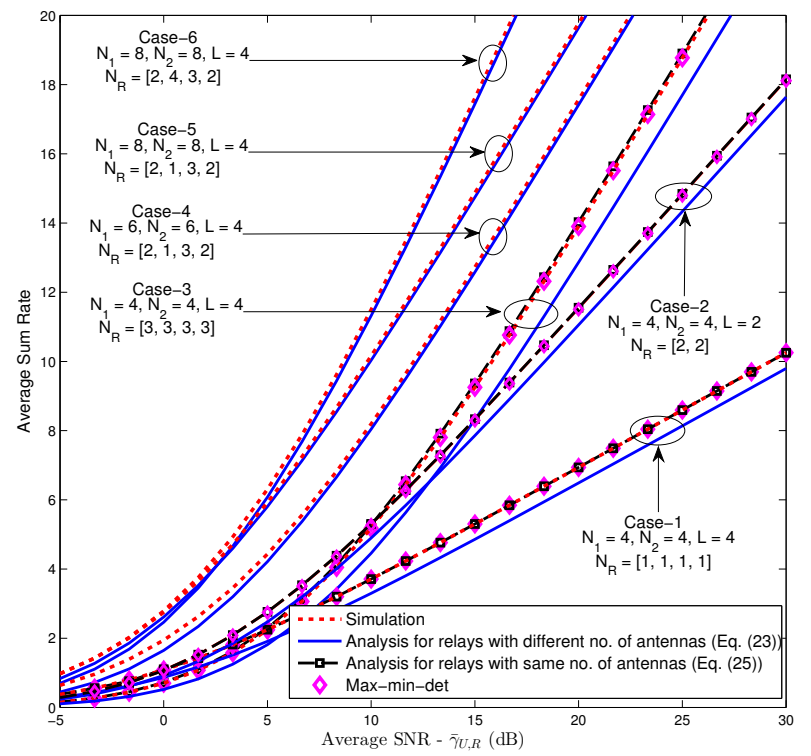

Fig. 1: The achievable average sum rate of the best relay selection. The average transmit SNRs $\bar{\gamma}_{i, l}$ and $\bar{\gamma}_{l, i}$ for $i \in\{1,2\}$ and $l \in\{1, \cdots, L\}$ are assumed equal and denoted as $\bar{\gamma}_{U, R}$. The hop distances are given by $d_{1, l}=d_{2, l}$, where $d_{i, l}$ is the distance between $R_{l}$ and $U_{i}$ for $l \in$ $\{1, \cdots, L\}$ and $i \in\{1,2\}$. The pathloss exponent is $\varpi=2.5$.

Further, the multinomial coefficient $\beta_{k, 2 l, N-N_{R}+1}$ in (25) can be written as

$\beta_{k, 2 l, N-N_{R}+1}=\sum_{i=k-N+N_{R}}^{k} \frac{\beta_{i, 2 l-1, N-N_{R}+1}}{(k-i) !} I_{\left[0,(2 l-1)\left(N-N_{R}\right)\right]}(i)$.

In (26), $\beta_{0,0, N-N_{R}+1}=\beta_{0,2 l, N-N_{R}+1}=1, \beta_{k, 1, N-N_{R}+1}=$ $1 / k !, \beta_{1,2 l, N-N_{R}+1}=2 l, I_{[a, c]}(b)=1$ for $a \leq b \leq c$, and $I_{[a, c]}(b)=0$ otherwise.

\section{Numerical Results}

In Fig. 1, the average sum rate of the best relay selection is investigated. Six different cases are plotted to obtain useful insights. The exact sum rate curves are plotted by using MonteCarlo simulations, while the sum rate approximations are plotted by using the analytical expressions in (23) and (25). Fig. 1 clearly reveals that the sum rate heavily depends on (i) the relay 
antenna count, (ii) user node antenna count, and (iii) number of relays. For example, at an SNR of $20 \mathrm{~dB}$, a quadruple-relay TWRN with quadruple-antenna user nodes (case-3) achieves a sum rate increase of seven bits/channel-use/Hz by upgrading the single-antenna relays (case-1) to triple-antenna relays (case-3). Moreover, at an SNR of $15 \mathrm{~dB}$, for the quadruple-relay TWRN, a sum rate gain of 2 bits/channel-use $/ \mathrm{Hz}$ can be achieved by increasing the number of user antennas from six (case-4) to eight (case-5). Besides, at an average SNR of $20 \mathrm{~dB}$, the quadruplerelay TWRN with quadruple-antenna users (case-3) provides about a sum rate gain of 2.5 bits/channel-use/Hz over the dualantenna/dual-relay counterpart (case-2). Fig. 1 shows that the sum rate curves obtained via relay selection based on maximizing the minimum determinant of the Wishart matrices (19) coincides with the simulated sum rate curves. Further, the two sets of analytical sum rate approximations are significantly tight to the simulated sum rate curves in their respective relay antenna configurations ${ }^{3}$.

\section{Conclusion}

For MIMO AF TWRNs with spatial multiplexing, this paper proposed and analyzed a novel relay selection strategy. It maximizes the sum rate by selecting the relay which maximizes the minimum of the determinant of the related Wishart matrices. While the exact derivation of the ergodic sum rate appears mathematically intractable, we derived the closed-form average sum rate approximations. Numerical results were presented to compare the performance gains of the proposed relay selection strategy and to validate our analysis. Our results reveal that the proposed relay selection strategy provides substantial sum rate improvements. For instance, sum rate gains in the order of 2 bits/channel-use/Hz or are achievable. Thus, boosting the throughput of MIMO TWRNs operating in wireless channels with rich scattering, and consequently prioritizing the use of available degrees of freedom for spatial multiplexing, is feasible.

\section{APPENDIX A}

\section{PROOF OF THE AVERAGE SUM RATE FOR RELAYS WITH DIFFERENT ANTENNA ARRAY SIZES}

In this appendix, the proof of the average sum rate is sketched when the relays are equipped with different number of antennas. To begin with, we recall the definition of the average sum rate approximation (21) as follows:

$$
\overline{\mathcal{R}}_{L^{*}} \approx \mathcal{E}_{\gamma_{\mathrm{eq}}}\left\{\left(\max _{l \in\{1, \cdots, L\}}\left(N_{R_{l}}\right)\right) \log \left(1+\gamma_{\mathrm{eq}}\right)\right\},
$$

where $\gamma_{\text {eq }}$ is the equivalent SNR and is given by

$$
\gamma_{\mathrm{eq}}=\min \left(\gamma_{U_{1, L^{*}}^{\min }}, \gamma_{U_{2, L^{*}}^{\min }}\right)
$$

Next, an approximation of the CDF of $\gamma_{\text {eq }}$ can be derived by using (12) as follows:

\footnotetext{
${ }^{3}$ The analytical sum rate curves (23) corresponding to case-1, case-2, and case- 3 slightly deviate away from the exact sum rate curve. Thus, this observation clearly justifies remark IV-2, and hence, the derivation of a tighter sum rate approximation (25) for the TWRNs with relays having the same number of antennas.
}

$$
F_{\gamma_{\mathrm{eq}}}(x) \approx 1-\prod_{i=1}^{2}\left(\frac{\Gamma\left(N_{i}-N_{R}^{\max }+1, \frac{\zeta_{i, L^{*} x}}{\alpha_{i, L^{*}-\beta_{i, L^{*} x}}}\right)}{\Gamma\left(N_{i}-N_{R}^{\max }+1\right)}\right),
$$

where $x \leq \min \left(\frac{\alpha_{2, L^{*}}}{\beta_{2, L^{*}}}, \frac{\alpha_{2, L^{*}}}{\beta_{2, L^{*}}}\right)$. Further, $F_{\gamma_{\mathrm{eq}}}(x)=1$ for $x>\min \left(\alpha_{1, L^{*}} / \beta_{1, L^{*}}, \alpha_{2, L^{*}} / \beta_{2, L^{*}}\right)$. Next, by using [17, Eq. (8.352.2)], (29) can be further expanded as

$$
\begin{aligned}
& F_{\gamma_{\mathrm{eq}}}(x) \approx 1-\exp \left(-\frac{\zeta_{1, L^{*}} x}{\alpha_{1, L^{*}}-\beta_{1, L^{*}} x}-\frac{\zeta_{2, L^{*}} x}{\alpha_{2, L^{*}}-\beta_{2, L^{*}} x}\right) \\
& \times \sum_{m=1}^{N_{1}-N_{R}^{\max }} \sum_{n=0}^{N_{2}-N_{R}^{\max }} \frac{1}{m ! n !}\left(\frac{\zeta_{1, L^{*}} x}{\alpha_{1, L^{*}}-\beta_{1, L^{*}} x}\right)^{m}\left(\frac{\zeta_{2, L^{*}} x}{\alpha_{2, L^{*}}-\beta_{2, L^{*}} x}\right)^{n}
\end{aligned}
$$

The CDF in (30) can further be simplified, whenever both the user nodes are equipped with the same number of antennas (i.e., $N_{1}=N_{2}=N$ ), and all transmit and noise powers at each relay are the same, as follows:

$$
F_{\gamma_{\mathrm{eq}}}(x) \approx 1-\mathrm{e}^{\frac{-2 \zeta_{L^{*} x}}{\alpha_{L^{*}-\beta_{L^{*}}}}} \sum_{m=0}^{N-N_{R}^{\max } N} \sum_{n=0}^{N-N_{R}^{\max }} \frac{1}{m ! n !}\left(\frac{\zeta_{L^{*}} x}{\alpha_{L^{*}}-\beta_{L^{*}} x}\right)^{m+n}
$$

where $\alpha_{L^{*}}=\alpha_{i, L^{*}}, \beta_{L^{*}}=\beta_{i, L^{*}}$, and $\zeta_{L^{*}}=\zeta_{i, L^{*}}$ for $i \in\{1,2\}$, and $x<\alpha_{L^{*}} / \beta_{L^{*}}$. By differentiating (31), an approximation of the PDF of $\gamma_{\text {eq }}$ can be derived as follows:

$$
\begin{aligned}
& f_{\gamma_{\mathrm{eq}}}(x) \approx \sum_{m=0}^{N-N_{R}^{\max }} \sum_{n=0}^{N-N_{R}^{\max }} \frac{\alpha_{L^{*}} \zeta_{L^{*}}^{m+n} x^{m+n-1}}{m ! n !\left(\alpha_{L^{*}}-\beta_{L^{*}} x\right)^{m+n+1}} \\
& \times\left(\frac{2 \zeta_{L^{*}} x}{\alpha_{L^{*}}-\beta_{L^{*}} x}-(m+n)\right) \exp \left(-\frac{2 \zeta_{L^{*}} x}{\alpha_{L^{*}}-\beta_{L^{*}} x}\right),
\end{aligned}
$$

where $x \leq \alpha_{L^{*}} / \beta_{L^{*}}$. Further, $f_{\gamma_{\mathrm{eq}}}(x)=0$ for $x \geq \alpha_{L^{*}} / \beta_{L^{*}}$. Next, an approximation of the ergodic sum rate can be derived by averaging the sum rate in (21) over the PDF of $\gamma_{\text {eq }}$ in (32) as

$$
\overline{\mathcal{R}}_{L^{*}} \approx \mathcal{E}\left\{\mathcal{R}_{L^{*}}\right\}=\frac{N_{R}^{\max }}{\ln (2)} \int_{0}^{\infty} \ln (1+x) f_{\gamma_{\mathrm{eq}}}(x) \mathrm{d} x .
$$

By substituting (32) into (33), the ergodic sum rate lower bound can be written in an integral form as follows:

$$
\overline{\mathcal{R}}_{L^{*}} \approx \frac{N_{R}^{\max }}{\ln (2)} \sum_{m=0}^{N-N_{R}^{\max }} \sum_{n=0}^{N-N_{R}^{\max }} \frac{1}{m ! n !}\left(\mathbb{I}_{1}-\mathbb{I}_{2}\right),
$$

where $\mathbb{I}_{1}$ and $\mathbb{I}_{2}$ can be defined as follows:

$$
\begin{aligned}
& \mathbb{I}_{1}=\frac{1}{2^{m+n}} \int_{0}^{\infty} t^{m+n} \mathrm{e}^{-t} \ln \left(\frac{2 \zeta_{L^{*}}+\left(\alpha_{L^{*}}+\beta_{L^{*}}\right) t}{2 \zeta_{L^{*}}+\beta_{L^{*}} t}\right) \mathrm{d} t \\
& \mathbb{I}_{2}=\frac{m+n}{2^{m+n}} \int_{0}^{\infty} t^{m+n-1} \mathrm{e}^{-t} \ln \left(\frac{2 \zeta_{L^{*}}+\left(\alpha_{L^{*}}+\beta_{L^{*}}\right) t}{2 \zeta_{L^{*}}+\beta_{L^{*}} t}\right) \mathrm{d} t .
\end{aligned}
$$

Next, $\mathbb{I}_{1}$ and $\mathbb{I}_{2}$ in (34b) and (34c), respectively, can be solved in closed-form by using [17, Eq. (4.337.5)], and the average sum rate approximation can be written as shown in (23).

\section{APPENDIX B}

PROOF OF THE AVERAGE SUM RATE FOR RELAYS WITH SAME ANTENNA ARRAY SIZE

In this appendix, the proof of the average sum rate approximation for the case of equal number of antennas at each relay 
is sketched. To begin with, the effective SNR (28) can be alternatively approximated as follows:

$$
\gamma_{\mathrm{eq}} \approx \gamma_{\mathrm{eq}}=\max _{l \in\{1, \cdots, L\}}\left(\min \left(\gamma_{U_{1, l}^{\min }}^{\mathrm{ub}}, \gamma_{U_{2, l}^{\min }}^{\mathrm{ub}}\right)\right),
$$

where $\gamma_{U_{i, l}^{\min }}^{\mathrm{ub}}$ for $i \in\{1,2\}$ is defined in (11). The CDF of $\gamma_{\mathrm{eq}}$ can be derived as follows:

$$
F_{\gamma_{\text {eq }}}(x)=\prod_{l=1}^{L}\left(1-\bar{F}_{\gamma_{U_{1, l}^{\text {min }}}^{\text {ub }}}(x) \bar{F}_{\gamma_{U_{2, l}}^{\text {ub }} \text { min }}(x)\right) .
$$

where $\bar{F}_{\gamma_{U_{i, l}^{\min }}^{\mathrm{ub}}}(x)$ is the complimentary CDF of $\gamma_{U_{i, l}^{\min }}^{\mathrm{ub}}$. By first substituting (12) into (36), and then by using [17, Eq. (8.352.2)], the CDF of $\gamma_{\mathrm{eq}_{1}}$ can be expanded as

$$
\begin{aligned}
& F_{\gamma_{\mathrm{eq}_{1}}}(x)=\prod_{l=1}^{L}\left(1-\exp \left(-\frac{\zeta_{1, l} x}{\alpha_{1, l}-\beta_{1, l} x}-\frac{\zeta_{2, l} x}{\alpha_{2, l}-\beta_{2, l} x}\right)\right. \\
& \left.\quad \times \sum_{m=1}^{N_{1}-N_{R}} \sum_{n=0}^{N_{2}-N_{R}} \frac{1}{m ! n !}\left(\frac{\zeta_{1, l} x}{\alpha_{1, l}-\beta_{1, l} x}\right)^{m}\left(\frac{\zeta_{2, l} x}{\alpha_{2, l}-\beta_{2, l} x}\right)^{n}\right),
\end{aligned}
$$

where $x<\min \left(\alpha_{1, l} / \beta_{1, l}, \alpha_{2, l} / \beta_{2, l}\right)$, and $F_{\gamma_{\mathrm{eq}}}(x)=1$ for $x \geq \min \left(\alpha_{1, l} / \beta_{1, l}, \alpha_{2, l} / \beta_{2, l}\right)$. The CDF in (37) can further be simplified whenever both the user nodes are equipped with the same number of antennas (i.e., $N_{1}=N_{2}=N$ ) as follows:

$F_{\gamma_{\mathrm{eq}_{1}}}(x)=\prod_{l=1}^{L}\left(1-\mathrm{e}^{\frac{-2 \zeta_{l} x}{\alpha_{l}-\beta_{l} x}} \sum_{m=1}^{N-N_{R}} \sum_{n=0}^{N-N_{R}} \frac{1}{m ! n !}\left(\frac{\zeta_{l} x}{\alpha_{l}-\beta_{l} x}\right)^{m+n}\right)$

where $x<\alpha_{l} / \beta_{l}, \alpha_{l}=\alpha_{1, l}=\alpha_{2, l}$, and $\beta_{l}=\beta_{1, l}=\beta_{2, l}$. Further, $F_{\gamma_{\text {eq }}}(x)=1$ for $x \geq \alpha_{l} / \beta_{l}$. The close-form derivation of the ergodic sum rate by using (38) appears mathematical intractable. Nevertheless, in the case of all transmit and noise powers at all the relays are the same, (38) can be written as

$$
F_{\gamma_{\mathrm{eq}_{1}}}(x)=\left(1-\mathrm{e}^{\frac{-2 \zeta x}{\alpha-\beta x}} \sum_{m=0}^{N-N_{R}} \sum_{n=0}^{N-N_{R}} \frac{1}{m ! n !}\left(\frac{\zeta x}{\alpha-\beta x}\right)^{m+n}\right)^{L} \text {, }
$$

where $\alpha_{l}=\alpha, \zeta_{l}=\zeta, \beta_{l}=\beta$ for $l \in\{1,2, \ldots L\}$, and $x<\alpha / \beta$. By first using the binomial expansion, and then by using [21, Eqn. (44)], the CDF of $\gamma_{\mathrm{eq}}$ can be expanded as

$$
\begin{aligned}
F_{\gamma_{\text {eq } 1}}(x)= & \sum_{l=0}^{L} \sum_{k=0}^{2 l\left(N-N_{R}\right)}(-1)^{l}\left(\begin{array}{l}
L \\
l
\end{array}\right) \beta_{k, 2 l, N-N_{R}+1}\left(\frac{\zeta x}{\alpha-\beta x}\right)^{k} \\
& \times \exp \left(-\frac{2 l \zeta x}{\alpha-\beta x}\right) \text { for } x<\frac{\alpha}{\beta},
\end{aligned}
$$

where the multinomial coefficient $\beta_{k, 2 l, N-N_{R}+1}$ is given by (26). By differentiating (40), the PDF of $\gamma_{\mathrm{eq}}$ can be derived as

$$
\begin{aligned}
f_{\gamma_{\text {eq } 1}}(x)= & \sum_{l=1}^{L} \sum_{k=0}^{2 l\left(N-N_{R}\right)}(-1)^{l}\left(\begin{array}{l}
L \\
l
\end{array}\right) \beta_{k, 2 l, N-N_{R}+1} \\
& \times\left(k-\frac{2 l \zeta x}{\alpha-\beta x}\right) \frac{\alpha \zeta^{k} x^{k-1}}{(\alpha-\beta x)^{k+1}} \exp \left(-\frac{2 l \zeta x}{\alpha-\beta x}\right),
\end{aligned}
$$

where $x<\alpha / \beta$. Further, $f_{\gamma_{\mathrm{eq}}}(x)=0$ for $x \geq \alpha / \beta$. Next, the approximation of the ergodic sum rate can be derived by averaging the sum rate in (21) over the PDF of $\gamma_{\mathrm{eq}}$ in (41) as

$$
\overline{\mathcal{R}}_{L^{*}} \approx \mathcal{E}\left\{\mathcal{R}_{L^{*}}\right\}=\frac{N_{R}}{\ln (2)} \int_{0}^{\infty} \ln (1+x) f_{\gamma_{\mathrm{eq}_{1}}}(x) \mathrm{d} x .
$$

Next, by using similar techniques to those used in deriving (23), an tight approximation to the ergodic sum rate, whenever all relays are equipped with the same number of antennas, can be derived as shown in (25).

\section{REFERENCES}

[1] B. Rankov and A. Wittneben, "Spectral efficient protocols for half-duplex fading relay channels," IEEE J. Sel. Areas Commun., vol. 25, no. 2, pp. 379-389, Feb. 2007.

[2] G. Amarasuriya, C. Tellambura, and M. Ardakani, "Two-way amplifyand-forward multiple-input multiple-output relay networks with antenna selection," IEEE J. Sel. Areas Commun., vol. 30, no. 8, pp. 1513-1529, Sep. 2012

[3] K. Loa et al., "IMT-advanced relay standards," IEEE Commun. Mag., vol. 48, no. 8, pp. 40-48, Aug. 2010.

[4] A. Paulraj, R. Nabar, and D. Gore, Introduction to space-time wireless communications, 1st ed. Cambridge, UK ; New York, NY : Cambridge University Press, 2003.

[5] L. Zheng and D. Tse, "Diversity and multiplexing: a fundamental tradeoff in multiple-antenna channels," IEEE Trans. Inf. Theory, vol. 49, no. 5, pp. 1073-1096, May 2003.

[6] G. Amarasuriya, C. Tellambura, and M. Ardakani, "Performance analysis of zero-forcing for two-way MIMO AF relay networks," IEEE Wireless Commun. Lett., vol. 1, no. 2, pp. 53-56, Apr. 2012.

[7] _ " "Joint beamforming and antenna selection for two-way amplify-andforward MIMO relay networks," in IEEE Int. Conf. on Commun. (ICC), Jun. 2012, pp. 4829-4834.

[8] A. Bletsas, A. Khisti, D. P. Reed, and A. Lippman, "A simple cooperative diversity method based on network path selection," IEEE J. Sel. Areas Commun., vol. 24, no. 3, pp. 659-672, Mar. 2006.

[9] Y. Zhao, R. Adve, and T. J. Lim, "Improving amplify-and-forward relay networks: optimal power allocation versus selection," IEEE Trans. Wireless Commun., vol. 6, no. 8, pp. 3114-3123, Aug. 2007.

[10] S. Atapattu, Y. Jing, H. Jiang, and C. Tellambura, "Relay selection schemes and performance analysis approximations for two-way networks," IEEE Trans. Commun., vol. 61, no. 3, pp. 987-998, Mar. 2013.

[11] H. Cui, M. Ma, L. Song, and B. Jiao, "Relay selection for two-way full duplex relay networks with amplify-and-forward protocol," IEEE Trans. Wireless Commun., vol. 13, no. 7, pp. 3768-3777, Jul. 2014.

[12] Y. Li, R. H. Y. Louie, and B. Vucetic, "Relay selection with network coding in two-way relay channels," IEEE Trans. Veh. Technol., vol. 59, no. 9, pp. 4489-4499, 2010

[13] L. Song, G. Hong, B. Jiao, and M. Debbah, "Joint relay selection and analog network coding using differential modulation in two-way relay channels," IEEE Trans. Veh. Technol., vol. 59, no. 6, pp. 2932-2939, Jul. 2010.

[14] I. Krikidis, "Relay selection for two-way relay channels with MABC DF: A diversity perspective," IEEE Trans. Veh. Technol., vol. 59, no. 9, pp. 46204628, Nov. 2010.

[15] Q. Zhou, Y. Li, F. Lau, and B. Vucetic, "Decode-and-forward two-way relaying with network coding and opportunistic relay selection," IEEE Trans. Commun., vol. 58, no. 11, pp. 3070-3076, Nov. 2010.

[16] T. Oechtering and H. Boche, "Bidirectional regenerative half-duplex relaying using relay selection," IEEE Trans. Wireless Commun., vol. 7, no. 5, pp. 1879-1888, May 2008.

[17] I. Gradshteyn and I. Ryzhik, Table of integrals, Series, and Products, 7th ed. Academic Press, 2007.

[18] J. Heath, R.W., S. Sandhu, and A. Paulraj, "Antenna selection for spatial multiplexing systems with linear receivers," IEEE Commun. Lett., vol. 5, no. 4, pp. 142-144, Apr. 2001.

[19] D. Gore, J. Heath, R.W., and A. Paulraj, "On performance of the zero forcing receiver in presence of transmit correlation," in IEEE Intl. Symp. on Inf. Theory (ISIT), Lausanne, Switzerland, Jul. 2002, pp. 1-2.

[20] E. F. Beckenbach and R. E. Bellman, Inequalities, 1st ed. Springer Verlag, 1965.

[21] A. Annamalai and C. Tellambura, "Error rates for Nakagami- $m$ fading multichannel reception of binary and M-ary signals," IEEE Trans. Commun., vol. 49, no. 1, pp. 58-68, Jan. 2001. 